\title{
A population-based cohort study of the risk of colorectal and other cancers among users of low-dose aspirin
}

\author{
S Friis*, ${ }^{*}$, HT Sørensen ${ }^{2,3}$, JK McLaughlin ${ }^{4,5}$, SP Johnsen ${ }^{2,6}$, WJ Blot ${ }^{4,5}$, and JH Olsen ${ }^{1,5}$ \\ 'Institute of Cancer Epidemiology, Danish Cancer Society, DK-2 100 Copenhagen, Denmark; ${ }^{2}$ Department of Clinical Epidemiology, Aarhus University \\ Hospital and Aalborg Hospital, DK-8600 Aarhus and DK-9100 Aalborg, Denmark; ${ }^{3}$ Department of Medicine V, Aarhus University Hospital, DK-8600 \\ Aarhus, Denmark; ${ }^{4}$ International Epidemiology Institute, Rockville, Maryland 20850, USA; ${ }^{5}$ Department of Medicine, Vanderbilt University Medical \\ Center, Vanderbilt-Ingram Comprehensive Cancer Center, Nashville, TN, USA; ${ }^{6}$ Department of Medicine M, Aalborg Hospital, DK-9I00 Aalborg, \\ Denmark
}

Using data from the population-based Prescription Database of North Jutland County and the Danish Cancer Registry, we compared cancer incidence among 29470 individuals prescribed low-dose aspirin at maximum doses of $150 \mathrm{mg}$ with expected incidence based on county-specific cancer rates, during a 9-year study period. We observed 238I cancer cases compared with 2187 expected, yielding a standardised incidence ratio (SIR) of 1.09 (95\% confidence interval $(\mathrm{Cl}), \mathrm{I} .05-\mathrm{I} . \mathrm{I}$ ). No apparent risk reductions were found for cancers of the colon (SIR, 0.9; $95 \% \mathrm{Cl}, 0.7-1.1$ ) or rectum (SIR, 1.0; 95\% Cl, 0.8- I.2), or for other site-specific cancers. Increased SIRs were observed for kidney cancer (SIR, I.4; 95\% Cl, I.I-1.7) and brain cancer (SIR, I.7; 95\% Cl, I.3-2.2), although the excess in the latter was confined to the first year of follow-up. Stratification by number of prescriptions and duration of follow-up revealed no apparent trends. The SIR for colorectal cancer was close to unity $(\mathrm{SIR}, 0.9 ; 95 \% \mathrm{Cl}, 0.6-1.2)$ among persons with 10 or more prescriptions who were followed for at least 5 years. Our results do not support a major protective effect of low-dose aspirin on the development of colorectal or other cancers. The observed excesses of kidney and brain cancers are not likely to be causally related to the use of low-dose aspirin.

British Journal of Cancer (2003) 88, 684-688. doi:10.1038/sj.bjc.6600760 www.bjcancer.com

(C) 2003 Cancer Research UK

Keywords: cancer; low-dose aspirin; risk; epidemiology; cohort study

Epidemiologic and experimental evidence strongly suggests that use of aspirin and other nonsteroidal anti-inflammatory drugs (NSAIDs) reduces the risk for colorectal cancer (Thun et al, 2002). A recent epidemiologic review (Garcia-Rodriguez and HuertaAlvarez, 2001) reported pooled relative risk estimates for colorectal cancer of 0.71 (95\% CI, $0.65-0.76)$ for use of aspirin and $0.63(95 \%$ CI, $0.57-0.70$ ) for use of nonaspirin NSAIDs. The extent to which aspirin and other NSAIDs protect against cancers other than colorectal cancer has not been established. There is some epidemiologic evidence that use of aspirin and other NSAIDs reduces the risk for cancers of the stomach and oesophagus (Thun et al, 1993; Farrow et al, 1998; Coogan et al, 2000), whereas the results are conflicting for nongastrointestinal cancers, including cancers of the lung (Thun et al, 1993; Schreinemachers and Everson, 1994; Langman et al, 2000), breast (Khuder and Mutgi, 2001; Cotterchio et al, 2001; Meier et al, 2002), prostate (Norrish et al, 1998; Langman et al, 2000; Leitzmann et al, 2002), bladder (Castelao et al, 2000; Langman et al, 2000), and ovary (Akhmedkhanov et al, 2001; Meier et al, 2002).

Only limited data are available on what dose and duration of treatment with aspirin and other NSAIDs are necessary to reduce the risks for colorectal and, potentially, other cancers. Some studies have evaluated the association between regular use of low-

*Correspondence: Dr S Friis; E-mail: friis@cancer.dk

Received 26 September 2002; revised 15 November 2002; accepted 15 November 2002 dose aspirin and colorectal cancer risk (Giovannucci et al, 1995; Rosenberg et al, 1998; Stürmer et al, 1998; Garcia-Rodriguez and Huerta-Alvarez, 2001), but the results are conflicting and little information has been provided for other cancer sites. Any substantial cancer-preventive effect of low-dose aspirin would have important public health implications. We have therefore examined the incidence of colorectal and other cancers in a Danish population-based cohort of persons receiving prescriptions for low-dose aspirin.

\section{MATERIAL AND METHODS}

The study was conducted within the population of North Jutland, a county with nearly 500000 inhabitants, representing approximately $9 \%$ of the total Danish population. Through a computerised accounting system maintained by Danish pharmacies, the taxsupported health insurance programme in Denmark refunds part of the costs of most drugs, including some over-the-counter medications, if prescribed by physicians. In North Jutland, this accounting system also provides prescription data to the Pharmacoepidemiologic Prescription Database (Gaist et al, 1997), initiated in 1989, which retains key data on prescriptions for refundable drugs dispensed from all pharmacies in the county. The data includes the type of drug prescribed according to the Anatomical Therapeutical Chemical (ATC) classification system (Capellá, 1993), tablet size, date of prescription, and the patient's civil registry number (a unique number assigned to all Danish 
residents that encodes gender and date of birth). The use of the civil registry number ensures that complete individual prescription histories can be established, and permits valid linkage of information between registers.

Through the Prescription Database, we identified 32794 individuals prescribed low-dose aspirin between 1 January 1989 and 31 December 1995. These prescriptions were identified by the ATC codes for aspirin (B01AC06 and N02BA01) in tablet sizes of 75,100 or $150 \mathrm{mg}$. All preparations of aspirin are available overthe-counter in Denmark, but low-dose aspirin, which is used almost exclusively for secondary prevention of cardiovascular disease, is generally prescribed by physicians, being reimbursable by $50 \%$ through the national health insurance programme. In Denmark, the recommended dose of low-dose aspirin for secondary prevention of cardiovascular disease is $75-150 \mathrm{mg}$ once daily, and the preparation is mainly prescribed in packets for 3month use (Sørensen et al, 2000).

Overall, $668(2.0 \%)$ of the identified persons prescribed low-dose aspirin were excluded because of (i) residency outside the county of North Jutland at the date of prescription $(n=617)$; (ii) an invalid civil registry number $(n=15)$; (iii) death prior to or at the date of prescription $(n=19)$; or (iv) parent (of patient) registered as customer $(n=17)$. After these exclusions, $32126(98.0 \%)$ persons were left for subsequent record linkage.

Information on cancer occurrence was obtained by linkage to the Danish Cancer Registry, which has recorded incident cases of cancer on a nationwide basis since 1943 (Storm et al, 1997). Cancers were classified according to a modified Danish version of the International Classification of Diseases, 7 th revision (Storm et al, 1997). Study subjects with a cancer diagnosis, except nonmelanoma skin cancer, prior to the date of first recorded prescription for low-dose aspirin $(n=2656 ; 8.1 \%)$ were excluded, leaving a final study cohort of 29470 (89.9\%) individuals.

The observation period for cancer began on the date of the first recorded prescription for low-dose aspirin and ended on the date of first primary cancer diagnosis except nonmelanoma skin cancer $(n=1962)$, date of death $(n=8493)$, or 31 December 1997 $(n=19015)$, whichever occurred first. Information on date of death was obtained through linkage to the National Mortality Files (Juel and Helweg-Larsen, 1999). The observed number of cancer cases among the cohort members was compared with the number expected based on cancer incidence rates among the general population of North Jutland. To obtain expected numbers, countyspecific incidence rates of first primary cancers, calculated by sex, 5 -year age groups, and 5-year calendar periods, were applied to the corresponding person-years of the cohort members. The standardised incidence ratio (SIR), that is, the ratio of the observed to the expected number of cancer cases, and $95 \%$ confidence intervals (CIs) were calculated for total cancer and for specific sites based on the assumption that the observed cancer cases followed a Poisson distribution (Bailar and Ederer, 1964). We also computed SIRs stratified by the number of low-dose aspirin prescriptions and by years of follow-up. For these analyses, each study subject contributed person-years to one to four categories of prescription frequency $(1 ; 2-4 ; 5-9$; or $\geqslant 10$ prescriptions), with follow-up for cancer $(<1 ; 1-4 ; 5-9$ years) beginning on the date of the first prescription within the given category of prescription frequency. A test for linear trend was used to evaluate trends in SIRs with the number of low-dose aspirin prescriptions or years of follow-up.

\section{RESULTS}

Characteristics of the study cohort of 15058 men (51\%) and 14412 women (49\%) are presented in Table 1 . The mean age at cohort entry, that is, time of first recorded prescription for low-dose aspirin, was 70 years, with only $6 \%$ of the cohort being younger
Table I Characteristics of users of low-dose aspirin recorded in the Prescription Database of North Jutland County, Denmark, between 1989 and 1995

\begin{tabular}{lcr}
\hline Characteristic & No. & (\%) \\
\hline Total no. of patients & 29470 & 100 \\
Men & 15058 & 51 \\
Women & 14412 & 49 \\
& & \\
Age at entry ${ }^{\text {a }}$ (years) & 1751 & 6 \\
$<50$ & 11456 & 39 \\
$50-69$ & 16263 & 55 \\
$\geqslant 70$ & & \\
& 70 years & \\
Mean age at entry & & 37 \\
& & 31 \\
Year of entry & 11044 & 32 \\
$1989-199 \mid$ & 9000 & \\
$1992-1993$ & 9426 & 20 \\
$1994-1995$ & & 24 \\
Number of prescriptions & 5794 & 32 \\
1 & 7069 & \\
$2-4$ & $709 \mid$ & \\
$5-9$ & 9516 & \\
$\geqslant 10$ & &
\end{tabular}

${ }^{a}$ Date of first recorded prescription for low-dose aspirin.

than 50 years at entry. The cohort members accrued a total of 121562 person-years, with an average length of follow-up since the first recorded prescription for low-dose aspirin of 4.1 years (range, $0-9$ years); $80 \%$ of subjects received two or more prescriptions for low-dose aspirin, and $32 \%$ received 10 or more prescriptions.

Overall, we observed 2381 cancer cases compared with 2187 expected, yielding a SIR of 1.09 (95\% CI, 1.05-1.13) (Table 2). No apparent risk reductions were observed for cancers of the gastrointestinal tract, including cancers of the colon (SIR, 0.9; 95\% CI, $0.7-1.1$ ), rectum (SIR, 1.0; 95\% CI, $0.8-1.2$ ), and stomach (SIR, 1.1; 95\% CI, 0.8-1.3). Increased SIRs were found for cancers of the kidney (SIR, 1.4; 95\% CI, 1.1-1.7) and brain (SIR, 1.7; 95\% $\mathrm{CI}, 1.3-2.2$ ). Slight risk elevations were seen for several smokingrelated cancers, including cancers of the buccal cavity and pharynx (SIR, 1.3; 95\% CI, 0.9 - 1.7), urinary bladder (SIR, 1.2; 95\% CI, 1.0 1.4), oesophagus (SIR, 1.3; 95\% CI, $0.9-1.9$ ), larynx (SIR, 1.5; $95 \%$ CI, $0.9-2.4$ ), and leukaemia (SIR, 1.3; 95\% CI, 1.0-1.6), whereas the SIR for lung cancer was close to expectation (SIR, 1.1; 95\% CI, $0.9-1.2)$. The SIR estimates for site-specific cancers were similar in men and women, except for cancer of the kidney (SIR, $1.6(95 \% \mathrm{CI}$, $1.1-2.1)$ in men vs 1.1 (95\% CI, 0.7-1.6) in women) and nonHodgkin's lymphoma (SIR, 1.4 (95\% CI, 1.0-1.9) in men vs 0.9 (95\% CI, 0.5-1.4) in women).

The SIRs did not vary markedly when stratified by age. The SIR for total cancer was 1.14 (95\% CI, 1.07-1.22) among persons below 70 years at cohort entry and 1.06 (95\% CI, 1.01-1.11) among older persons (data not shown). Similarly, for specific cancer sites, including colorectal cancer and kidney cancer, the SIR estimates did not differ substantially between these two age groups.

Table 3 presents SIR estimates, stratified by number of prescriptions for low-dose aspirin and years of follow-up, for total cancer, selected gastrointestinal cancers, and kidney cancer. Overall, stratification by duration of follow-up yielded SIRs for total cancer of $1.2(95 \% \mathrm{CI}, 1.1-1.3)$ for less than 1 year of followup, 1.1 (95\% CI, 1.0 - 1.1) for 1-4 years of follow-up, and $1.1(95 \%$ CI, 1.0-1.2) for 5-9 years of follow-up. Similar SIRs were observed for total cancer in the four categories of prescription frequency. The SIRs for colorectal and stomach cancer decreased slightly with 
Table 2 Standardised incidence ratios (SIR) and 95\% confidence intervals (CI) for cancers at selected sites, stratified by sex, among users of low-dose aspirin in North Jutland county, Denmark, 1989-1997

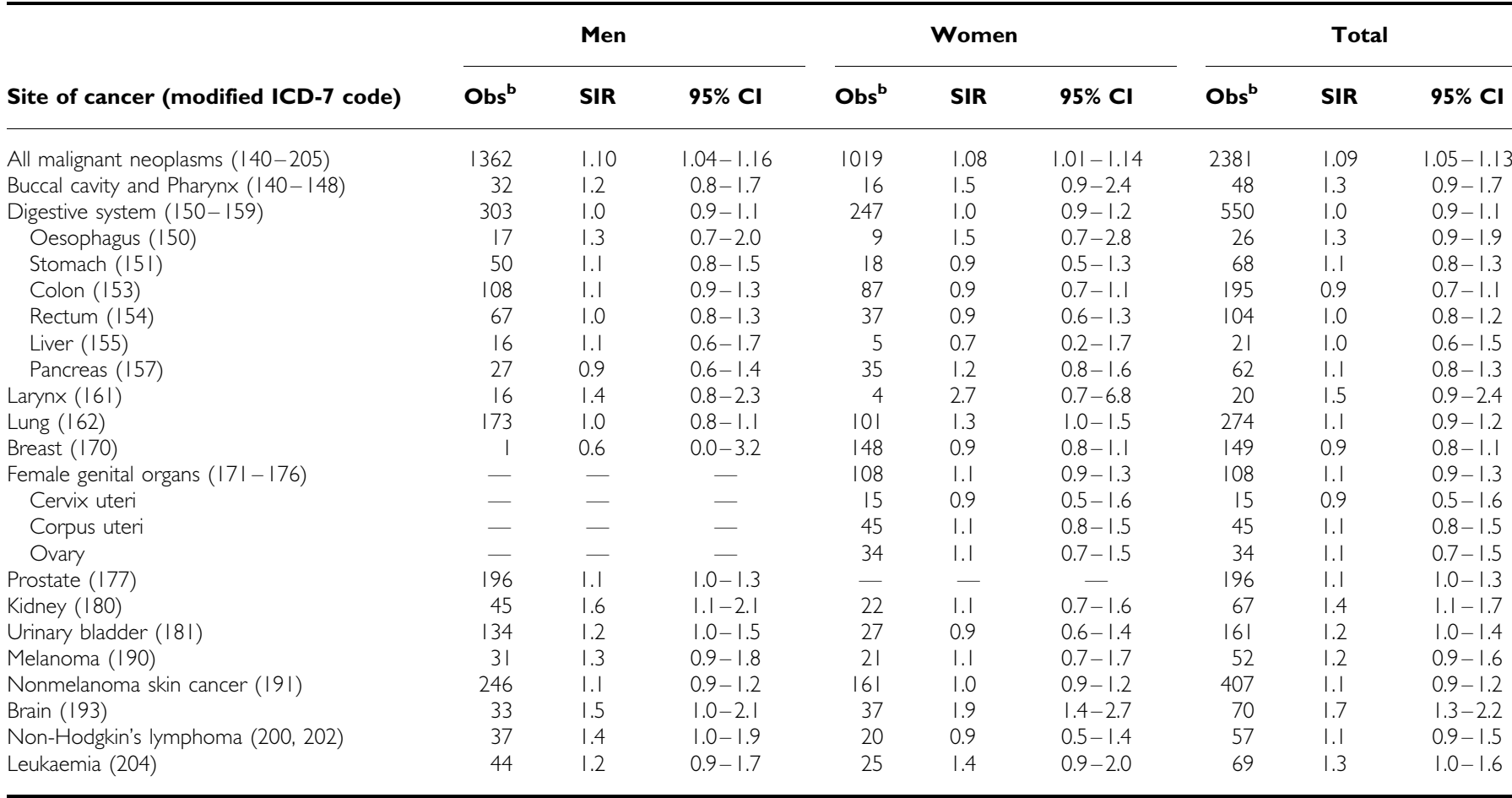

${ }^{\mathrm{a}}$ Person-years of observation in each stratum: men, 61 093; women, 60469 . ${ }^{\mathrm{b}}$ Observed number of cases.

both increasing number of prescriptions and increasing years of follow-up, but none of the trends was statistically significant. No consistent trends in risk estimates were observed for cancers of the oesophagus or kidney. Among persons with 10 or more prescriptions, the SIR for kidney cancer was of the same magnitude in the 1- to 4-year (SIR, 1.6; 95\% CI, 0.7-3.1) and 5- to 9-year (SIR, 1.7; 95\% CI, 0.9-2.9) follow-up periods.

The overall increased SIR for brain cancer was entirely due to an excess in the first year of follow-up (SIR, 4.7; 95\% CI, 3.4-6.3) among persons receiving one prescription (SIR, 6.2; 95\% CI, 4.1 9.1) or 2-4 prescriptions (SIR, 3.5; 95\% CI, 2.0-5.7) (data not shown). For the remaining sites of interest, including cancers of the lung, breast, prostate, bladder, and ovary, stratification by number of prescriptions and years of follow-up revealed no consistent trends (data not shown).

\section{DISCUSSION}

In this population-based cohort study, we found little evidence of a reduced risk for colorectal or other cancers among nearly 30000 individuals receiving prescriptions for low-dose aspirin. There was a slight decreasing trend in risks for colorectal and stomach cancers with increasing number of prescriptions and length of follow-up, however, the SIRs for both cancers were close to unity among persons with 10 or more prescriptions (equivalent to an estimated period of aspirin therapy of minimum 30 months) who were followed for at least 5 years.

Our results are consistent with those of two previous studies reporting on colorectal cancer risk among users of low-dose aspirin. In the only randomised clinical trial of aspirin and cancer risk published to date (Stürmer et al, 1998), based on the Physicians' Health Study in the United States, both randomised (5 years of follow-up) and post-trial observational analyses (up to 12 years of follow-up) revealed no association between the assigned low dose of aspirin (325 mg every other day) and risk for colorectal cancer. Limitations however, include possible exposure misclassification in the post-trial analysis (exposure was assessed only at the beginning of the observational follow-up period) and limited statistical power. A large nested case - control study (Garcia-Rodriguez and Huerta-Alvarez, 2001), based on the General Practice Research Database in the UK, reported a significant reduction in risk for colorectal cancer (OR, 0.6; 95\% CI, 0.4-0.9) associated with the current use of aspirin at daily doses of $300 \mathrm{mg}$ or greater for at least 6 months, whereas daily doses of 75 and $150 \mathrm{mg}$ aspirin were not associated with a reduced risk. A limitation of that study was the relatively small number of subjects with long continuous ( $>2$ years) exposure to aspirin. In contrast, a population-based case - control study (Rosenberg et al, 1998) and a cohort analysis based on the Nurses' Health Study (Giovannucci et al, 1995) reported that regular users of low-dose aspirin had a substantially reduced risk for colorectal cancer. However, the doses examined in the latter study had upper limits intermediate between cardioprotective and anti-inflammatory doses, and no results were presented separately for low-dose aspirin as defined in the present study.

The strengths of our study are the population-based design, the continuously updated data on aspirin use based on a prescription database covering all pharmacies in a Danish county, the relatively large number of outcomes, and the complete follow-up obtained by use of the unique civil registry number and computerised linkage to the Danish Cancer Registry. The Cancer Registry covers the entire population of Denmark and has been shown to have accurate and virtually complete ascertainment of cancer cases (Storm et al, 1997). Although low-dose aspirin is available over the counter, a $50 \%$ government refund at the time of prescription exists and is used predominantly for conditions requiring physician attendance; thus, we have probably identified most of the patients using low-dose aspirin on a regular basis in the North Jutland population during the period of registration. 
Table 3 Standardised incidence ratios (SIR) and 95\% confidence intervals (Cl) for selected cancer sites among users of low-dose aspirin, stratified by number of prescriptions and years of follow-up, North Jutland county, Denmark, 1989- 1997

Follow-up (years)

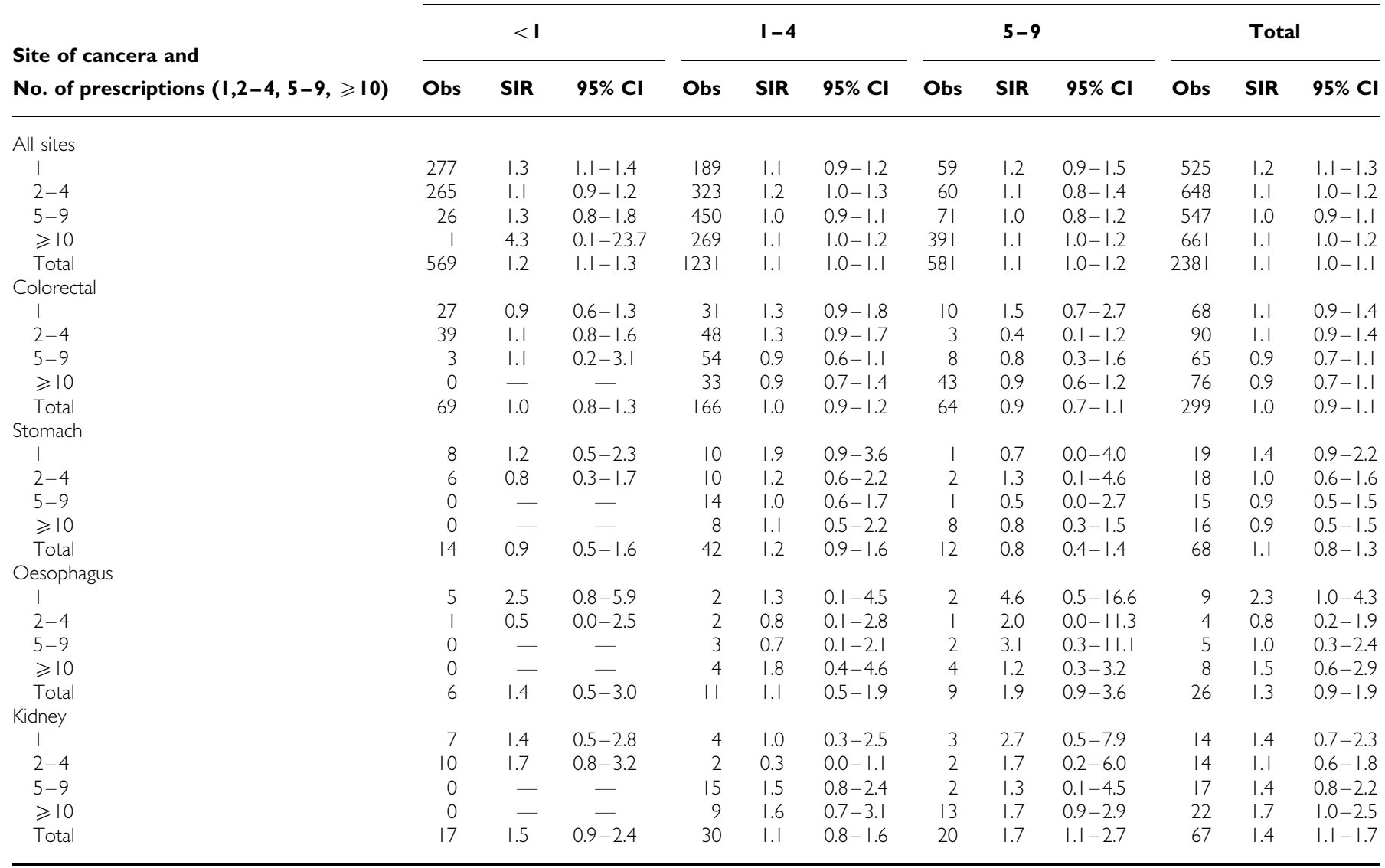

In our register-based approach, we had to rely on prescription data, with no information on indications, dose schedules, or degree of compliance. However, a large proportion of our cohort members can be assumed to have received low-dose aspirin for secondary prevention of cardiovascular disease at daily doses of 75-150 mg. Also, the fact that drug exposure was based on prescriptions actually dispensed at pharmacies and paid for in part by the patient implies high compliance. Nonetheless, some misclassification because of noncompliance is possible, leading to attenuation of the risk estimates.

Our study had a number of other limitations. We had no data on over-the-counter purchase of high-dose aspirin. Use patterns of high-dose aspirin and nonaspirin NSAIDs for analgesic or antiinflammatory purposes may have been different among persons prescribed low-dose aspirin than among the general population, thus introducing possible exposure misclassification of aspirin and/or confounding by nonaspirin NSAIDs. The finding of slightly increased SIRs for several smoking-related cancers is consistent with the fact that smoking is a strong independent risk factor for both ischaemic cardiovascular disease and cancer (Dreyer and Olsen, 1998), and that low-dose aspirin in Denmark is prescribed almost exclusively as secondary prevention in patients with cardiovascular disease. Our inability to adjust for concurrent NSAID use, smoking, and other potential confounders may have obscured a cancer-preventive effect of low-dose aspirin. Nevertheless, the absence of any substantial trends in SIRs with the number of prescriptions of low-dose aspirin or years of follow-up argues against any important inverse association between use of low-dose aspirin and cancer risk

The increased risk for kidney cancer may partly reflect an increased prevalence of hypertension among the study subjects with ischaemic heart disease. Patients with kidney tumours may present with hypertension some years prior to kidney cancer diagnosis (Steffens et al, 1992), and there is increasing evidence that hypertension per se increases the risk for kidney cancer (McLaughlin and Lipworth, 2000). Other arguments against a causal relation between the use of low-dose aspirin and kidney cancer are the gender difference and the absence of a dose response relation or temporal trend in the risk estimates for kidney cancer in this study, in addition to the largely null results from previous epidemiological studies of aspirin intake and kidney cancer (McLaughlin and Lipworth, 2000).

The increased risk observed for brain cancer is likely to be a result of 'reverse causation' (i.e. prescription for low-dose aspirin to treat symptoms of brain cancer). The excess risk was confined exclusively to the first year of follow-up among patients who received only a few prescriptions for low-dose aspirin. Some patients with brain tumours may have received anti-thrombotic treatment with low-dose aspirin prior to diagnosis, because they presented with symptoms resembling thrombotic cerebral diseases, for example, transient ischaemic attacks.

The duration of exposure to aspirin necessary to prevent colorectal or other cancers has not been firmly established. In one study, a protective effect at doses of at least $300 \mathrm{mg}$ daily became evident after only 6 months of continuous treatment (Garcia- 
Rodriguez and Huerta-Alvarez, 2001), but other studies have suggested that longer periods of treatment are necessary to achieve a protective effect. Our observation period of up to 9 years with an average follow-up of 4.1 years may be too short to detect a cancerpreventive effect, even if the effect is on disease progression. However, a substantial proportion of the patients were likely to be prevalent users at the start of the registration period and therefore to have had exposure before the observation period.

In summary, patients prescribed low-dose aspirin at doses of maximum $150 \mathrm{mg}$ were not at a substantially reduced risk for colorectal or other cancers. The available evidence so far does not support a major protective effect of low-dose aspirin on the development of colorectal or other cancers. Further studies are needed to establish what dose and duration of treatment by aspirin and other NSAIDs are necessary to prevent colorectal and possibly other cancers.

\section{ACKNOWLEDGEMENTS}

The study was funded by the Danish Cancer Society (Grant no. 99 100 09). The activities at the Institute of Cancer Epidemiology were supported by The International Epidemiology Institute. We thank Lars Thomassen for help with the data management.

\section{REFERENCES}

Akhmedkhanov A, Toniolo P, Zeleniuch-Jacquotte A, Kato I, Koenig KL, Shore RE (2001) Aspirin and epithelial ovarian cancer. Prev Med 33: $682-687$

Bailar JC, Ederer F (1964) Significance factors for the ratio of a Poisson variable to its expectation. Biometrics 20: $639-643$

Capellá D (1993) Descriptive tools and analysis. In Drug Utilization Studies: Methods and Uses. WHO Regional Publications (European Series No. 45), Dukes MNG (ed) pp 55-78. Geneva: World Health Organization

Castelao JE, Yuan JM, Gago-Dominguez M, Yu MC, Ross RK (2000) Nonsteroidal anti-inflammatory drugs and bladder cancer prevention. $\mathrm{Br} J$ Cancer 82: $1364-1369$

Coogan PF, Rosenberg L, Palmer JR, Strom BL, Zauber AG, Stolley PD, Shapiro S (2000) Nonsteroidal anti-inflammatory drugs and risk of digestive cancers at sites other than the large bowel. Cancer Epidemiol Biomarkers Prev 9: 119-123

Cotterchio M, Kreiger N, Sloan M, Steingart A (2001) Nonsteroidal antiinflammatory drug use and breast cancer risk. Cancer Epidemiol Biomarkers Prev 10: 1213-1217

Dreyer L, Olsen JH (1998) Cancer risk of patients discharged with acute myocardial infarct. Epidemiology 9: 178-183

Farrow DC, Vaughan TL, Hansten PD, Standford JL, Risch HA, Gammon MD, Chow WH, Dubrow R, Ahsan H, Mayne ST, Schoenberg JB, West AB, Rotterdam H, Fraumeni JF Jr, Blot WJ (1998) Use of aspirin and other nonsteroidal anti-inflammatory drugs and risk of esophageal and gastric cancer. Cancer Epidemiol Biomarkers Prev 7: 97-102

Gaist D, Sørensen HT, Hallas J (1997) The Danish prescription registries. Dan Med Bull 44: 445 -448

Garcia-Rodriguez LA, Huerta-Alvarez C (2001) Reduced risk of colorectal cancer among long-term users of aspirin and nonaspirin nonsteroidal anti-inflammatory drugs. Epidemiology 12: 88-93

Giovannucci E, Egan KM, Hunter DJ, Stampfer MJ, Colditz GA, Willett WC, Speizer FE (1995) Aspirin and the risk of colorectal cancer in women. $N$ Engl J Med 333: 609-614

Juel K, Helweg-Larsen K (1999) The Danish registers of causes of death. Dan Med Bull 46: $354-357$

Khuder SA, Mutgi AB (2001) Breast cancer and NSAID use: a meta-analysis. Br J Cancer 84: $1188-1192$

Langman MJ, Cheng KK, Gilman EA, Lancashire RJ (2000) Effect of antiinflammatory drugs on overall risk of common cancer: case - control study in general practice research database. BMJ 320: $1642-1646$

Leitzmann MF, Stampfer MJ, Ma J, Chan JM, Colditz GA, Willett WC, Giovannucci E (2002) Aspirin use in relation to risk of prostate cancer. Cancer Epidemiol Biomarkers Prev 11: 1108-1111

McLaughlin JK, Lipworth L (2000) Epidemiologic aspects of renal cell cancer. Semin Oncol 27: 115-123

Meier CR, Schmitz S, Jick H (2002) Association between acetaminophen or nonsteroidal anti-inflammatory drugs and risk of developing ovarian, breast, or colon cancer. Pharmacotherapy 22: 303-309

Norrish AE, Jackson RT, McRae CU (1998) Non-steroidal anti-inflammatory drugs and prostate cancer progression. Int J Cancer 77: $511-515$

Rosenberg L, Louik C, Shapiro S (1998) Nonsteroidal anti-inflammatory drug use and reduced risk of large bowel carcinoma. Cancer 82: $2326-$ 2333

Schreinemachers DM, Everson RB (1994) Aspirin use and lung, colon, and breast cancer incidence in a prospective study. Epidemiology 5: 138-146

Sørensen HT, Mellemkjær L, Blot WJ, Nielsen GL, Steffensen FH, McLaughlin JK, Olsen JH (2000) Risk of upper gastrointestinal bleeding associated with use of low-dose aspirin. Am J Gastroenterol 95: 2218 2224

Steffens J, Bock R, Braedel HU, Isenberg E, Buhrle CP, Ziegler M (1992) Renin-producing renal cell carcinomas - clinical and experimental investigations on a special form of renal hypertension. Urol Res 20: 111 115

Storm HH, Michelsen EV, Clemmensen IH, Pihl J (1997) The Danish Cancer Registry - history, content, quality and use. Dan Med Bull 44: 535-539

Stürmer T, Glynn RJ, Lee IM, Manson JE, Buring JE, Hennekens CH (1998) Aspirin use and colorectal cancer: post-trial follow-up data from the Physicians' Health Study. Ann Intern Med 128: 713-720

Thun MJ, Henley SJ, Patrono C (2002) Nonsteroidal anti-inflammatory drugs as anticancer agents: mechanistic, pharmacologic, and clinical issues. J Natl Cancer Inst 94: 252-266

Thun MJ, Namboodiri MM, Calle EE, Flanders WD, Heath CW Jr (1993) Aspirin use and risk of fatal cancer. Cancer Res 53: 1322-1327 